MATHEMATICS OF COMPUTATION

Volume 78, Number 265, January 2009, Pages 617-618

S 0025-5718(08)02175-3

Article electronically published on August 29, 2008

\title{
ERRATA TO "EVALUATION OF ZETA FUNCTION OF THE SIMPLEST CUBIC FIELD AT NEGATIVE ODD INTEGERS"
}

\author{
HYUN KWANG KIM
}

Theorem 3.2 in the paper is incorrect since the left-hand side of equation (15) in [2] is multiplicative while the right-hand side is not. Therefore, Theorem 5.2 and Table 1, which use the result of Theorem 3.2, are wrong. However, the description of a Siegel lattice (Theorem 4.4) is correct. From the description of a Siegel lattice, using the methods in [1, we can compute the values of $\zeta_{K}(-1)$ for the first twentyfive simplest cubic fields, and the values of $\zeta_{K}(-3)$ and $\zeta_{K}(-5)$ for the first ten simplest cubic fields.

TABLE 1. Values of $\zeta_{K}(-1)$ for the first twenty-five simplest cubic fields

\begin{tabular}{|c|c|c||c|c|c|}
\hline$m$ & $D$ & $-21 \zeta_{K}(-1)$ & $m$ & $D$ & $-21 \zeta_{K}(-1)$ \\
\hline-1 & 7 & 1 & 20 & $7 * 67$ & $3^{3} * 7 * 11^{2} * 13$ \\
\hline 1 & 13 & 7 & 22 & $13 * 43$ & $3^{3} * 7 * 43 * 61$ \\
\hline 2 & 19 & $3 * 7$ & 23 & 607 & $2^{2} * 7 * 23743$ \\
\hline 4 & 37 & $3 * 7^{2}$ & 25 & 709 & $2^{2} * 7 * 36229$ \\
\hline 7 & 79 & $7 * 199$ & 26 & $7 * 109$ & $2^{2} * 3 * 7 * 43 * 409$ \\
\hline 8 & 97 & $7 * 367$ & 28 & 877 & $7^{4} * 19 * 43$ \\
\hline 10 & 139 & $5^{2} * 7 * 43$ & 29 & 937 & $2^{2} * 3 * 7^{2} * 3931$ \\
\hline 11 & 163 & $2^{2} * 3 * 7 * 13^{2}$ & 31 & 1063 & $3 * 7^{2} * 79 * 337$ \\
\hline 13 & $7 * 31$ & $3^{2} * 7 * 13 * 37$ & 32 & 1129 & $7 * 37 * 15817$ \\
\hline 14 & $13 * 19$ & $3 * 7 * 19 * 109$ & 34 & $7 * 181$ & $3^{2} * 7 * 163 * 577$ \\
\hline 16 & 313 & $7^{2} * 2131$ & 35 & $13 * 103$ & $3^{2} * 7 * 111091$ \\
\hline 17 & 349 & $2^{2} * 7 * 43 * 103$ & 37 & 1489 & $7^{2} * 109 * 1951$ \\
\hline 19 & $7 * 61$ & $3 * 5^{2} * 7^{2} * 61$ & \multicolumn{3}{|}{} \\
\hline
\end{tabular}

Received by the editor January 23, 2008.

2000 Mathematics Subject Classification. Primary 11R42.

(C)2008 American Mathematical Society Reverts to public domain 28 years from publication 
TABLE 2. Values of $\zeta_{K}(-3)$ and $\zeta_{K}(-5)$ for the first ten simplest cubic fields

\begin{tabular}{|c|c|c|c|}
\hline$m$ & $D$ & $8190 \zeta_{K}(-3)$ & $-3591 \zeta_{K}(-5)$ \\
\hline-1 & 7 & $3^{2} * 337$ & $3 * 19 * 7393$ \\
\hline 1 & 13 & $3^{2} * 13^{2} * 151$ & $3 * 19 * 73 * 91807$ \\
\hline 2 & 19 & $3^{3} * 13 * 41 * 227$ & $3^{2} * 127 * 21720427$ \\
\hline 4 & 37 & $3^{3} * 7 * 1834999$ & $3^{2} * 19 * 109 * 2034277813$ \\
\hline 7 & 79 & $3^{2} * 349 * 22333261$ & $3 * 19^{2} * 7207 * 20423409133$ \\
\hline 8 & 97 & $3^{2} * 13 * 4363 * 578167$ & $3 * 7 * 19 * 3820580605391311$ \\
\hline 10 & 139 & $3^{2} * 5^{2} * 16275480877$ & $3 * 5^{2} * 19 * 55970747229303661$ \\
\hline 11 & 163 & $2^{2} * 3^{3} * 11 * 37 * 89 * 2868577$ & $2^{2} * 3^{2} * 7 * 19 * 31 * 241 * 2251 * 3259 * 1752943$ \\
\hline 13 & $7 * 31$ & $3^{5} * 7 * 229 * 212601511$ & $3^{3} * 19 * 619 * 80713 * 417756469213$ \\
\hline 14 & $13 * 19$ & $3^{3} * 5279 * 1437507551$ & $3^{2} * 19 * 283 * 919384681715200627$ \\
\hline
\end{tabular}

\section{REFERENCES}

[1] S.J. Cheon, H.K. Kim and J.H. Lee, Evaluation of the Dedekind zeta functions of some nonnormal cubic totally real cubic fields at negative odd integers, Manuscripta Math., 124 (2007), 551-560. MR 2357798

[2] H.K. Kim and J.S. Kim, Evaluation of zeta function of the simplest cubic field at negative odd integers, Mathematics of Computation, 71 (2002),1243-1262. MR:1898754 (2003h:11143)

Department of Mathematics, Pohang University of Science and Technology, Pohang 790-784, KoreA

E-mail address: hkkim@postech.ac.kr 